\title{
I. - MÉTHODES DE PRÉPARATION DES LOTS
}

\author{
J.-C. LASSERAN \\ avec la collaboration technique de M. BALlACEY, P. BARThélÉmY \\ G. Montagu et F. Villedey \\ Institut Technique des Céréales et des Fourrages, \\ 8 , avenue du Président Wilson, \\ Paris $16 \mathrm{e}$

\section{RÉSUMÉ}

Une étude ayant pour thème "l'influence du mode de conditionnement sur la qualité du maïs " a été entreprise en 1968. Six traitements ont été réalisés dans des conditions différentes, considérées comme bonnes ou mauvaises, mais bien déterminées.

Trois modes de préstockage du maïs humide ont été pratiqués avant séchage :

I Court séjour en cellule de réception, d'une durée inférieure à 30 heures, soit pratiquement un séchage immédiat après récolte $(R)$.

$2^{\circ}$ Séjour prolongé en cellule, d'une durée de 6 jours, ayant provoqué des échauffements spontanés (E).

$3^{\circ}$ Conservation en cellule pendant 45 jours par ventilation d'air refroidi au moyen $d^{\prime} u n$ groupe frigorifique (F).

Les opérations de séchage ont été réalisées en un seul passage dans un séchoir continu et aux deux températures suivantes:

- $80^{\circ} \mathrm{C}$ : température considérée comme modérée $(80)$;

- ${ }^{4} 40^{\circ} \mathrm{C}$ : température considérée comme excessive ( $\mathrm{I}_{4} \mathrm{O}$ ).

L'ensemble des mesures effectuées au cours de ces essais a permis l'établissement de données chiffrées relatives à la technique de séchage utilisée. Les principaux résultats qui ressortent de cette étude sont les suivants : le rendement de séchage, la consommation thermique massique, le temps de séchage et le débit du séchoir sont considérablement améliorés lorsque le séchage est effectué à haute température.

A la suite de ces traitements, les spécialistes de la commercialisation des grains et de l'utilisation du maïs en amidonnerie, les biochimistes, les microbiologistes et les zootechniciens donneront leur avis sur la qualité du maïs obtenu.

\section{INTRODUCTION}

L,e maïs est toujours récolté à une teneur en eau élevée. Il faut le sécher artificiellement aussi rapidement que possible pour assurer sa conservation. Mais un délai sépare obligatoirement l'instant de la récolte de celui du séchage. Son importance 
est fonction d'un très grand nombre de facteurs (Journ, I966 $a$; MAQUET, I966). Le séchage est à juste titre présenté comme un goulot d'étranglement dans les conditions existant actuellement en France au niveau des organismes stockeurs. Cette situation peut conduire à choisir des solutions d'équipement mal adaptées à une production de maïs :

I Une installation de séchage surdimensionnée absorbe immédiatement les apports massifs de grain, mais elle nécessite des investissements importants entrâ̂nant un coût d'amortissement élevé en raison de la réduction du temps de fonctionnement annuel.

$2^{0}$ Une installation de puissance insuffisante risque de provoquer une accumulation de grain humide difficile à résorber ; plusieurs jours, voire une semaine, peuvent s'écouler entre la récolte et le séchage. Certes, les coûts d'amortissement sont plus bas que dans le cas précédent, mais ils peuvent être compensés par des pertes de qualité dues, par exemple, à des échauffements (CALET, MERCIER, Ig66) lorsque l'organisme stockeur ne possède pas les moyens efficaces pour assurer un bon préstockage en cellule d'attente par une ventilation de maintien d'air frais ou refroidi (JouIN, I957, I965, I966 $a$ et $b$; MATTEI, I969 $a$ et $b$ ).

L'incidence de la température de séchage sur la valeur alimentaire et technologique du grain est mal connue : il n'existe pas de règle précise et la tendance actuelle des utilisateurs est d'augmenter la température jusqu'à 120 ou $130^{\circ} \mathrm{C}$ afin d'accroître le débit de leur séchoir. Signalons à cet égard que les études américaines d'HATHAWAY et al. (1952), CABELI et al. (1958), MILNER et WOODFORDE (I965) n'ont pas été entreprises à des températures dépassant $105^{\circ} \mathrm{C}$.

Par ailleurs, l'influence de l'interaction entre le délai d'attente et la température de séchage sur la qualité du maîs n'a encore jamais été étudiée.

Ces considérations nous ont conduit à comparer les effets de séchage soit immédiatement après récolte, soit après échauffements spontanés ou après un préstockage par ventilation d'air refroidi, en choisissant deux températures de séchage extrêmes.

\section{MATÉRIEL, ET MÉTHODES}

\section{I. - Culture et récolte du mais grain}

Le mais (variété INRA 258) a été cultivé en I968 dans un champ homogène de Io hectares et récolté à l'automne après élimination des 4 rangées périphériques au moyen d'un corn sheller automoteur à deux rangs.

La quantité totale de maïs-grain humide destinée à ces essais était de l'ordre de $54^{\circ}$ quintaux ; celle-ci a été transportée dans les meilleurs délais au C.N.E.E.M.A à Antony, pour y subir les traitements prévus dans le protocole expérimental. L'humidité moyenne à la récolte était de $36 \mathrm{p}$. Ioo par rapport à la substance fraîche.

\section{II. - Description des installations utilisées pour effectuer les traitements}

Le dispositif expérimental comprend les éléments suivants :

- une fosse de réception du grain humide;

- un appareil de nettoyage, type nettoyeur-trieur-séparateur;

- une manutention par bandes transporteuses et élévateurs à godets ;

- un ensemble de 9 cellules de stockage identiques disposées en carré de 3 cellules $\times 3$ cellules. 
Les caractéristiques de chacune d'elles sont les suivantes : section carrée de $2 \times 2$ mètres; hauteur moyenne de 3 mètres ; capacité de 12 mètres cubes; contenance de 90 quintaux environ. De plus, les cellules sont à fond pyramidal et munies de tubes antidynamiques pour faciliter la vidange. Une gaine de distribution d'air située sous une rangée de 3 cellules permet la ventilation d'une à trois cellules au moyen de registres mobiles; les ventilateurs de 2 rangées peuvent être alimentés avec de l'air refroidi par l'intermédiaire d'un générateur d'air froid, d'une puissance maxima de 24 ooo frigories à l'heure; les 6 cellules ainsi équipées sont thermiquement isolées par des plaques de polystyrène expansé de ro cm d'épaisseur. Enfin, une installation de silothermométrie par thermocouples permet de mesurer et d'enregistrer la température du grain en différents points des cellules.

- Un séchoir de type classique avec zone de séchage et zone de refroidissement (voir schéma de la figure I). Le séchoir possède une seule colonne dans laquelle le grain s'écoule par gravité ; ses dimensions sont les suivantes : $0,20 \mathrm{~m}$ d'épaisseur, 2,00 $\mathrm{m}$ de largeur, 3,00 $\mathrm{m}$ de hauteur, dont 2, $40 \mathrm{~m}$ correspondant à la zone de séchage et $0,60 \mathrm{~m}$ à la zone de refroidissement; le volume de la zone de séchage est de $0,96 \mathrm{~m}^{3}$; les parois de la colonne sont constituées de cadres grillagés démontables. L'air chaud est fourni par un générateur à échangeur thermique dont la puissance calorifique peut varier de Ioo ooo à 300 ooo $\mathrm{kcal} / \mathrm{h}$, assurant ainsi une température variable, mais

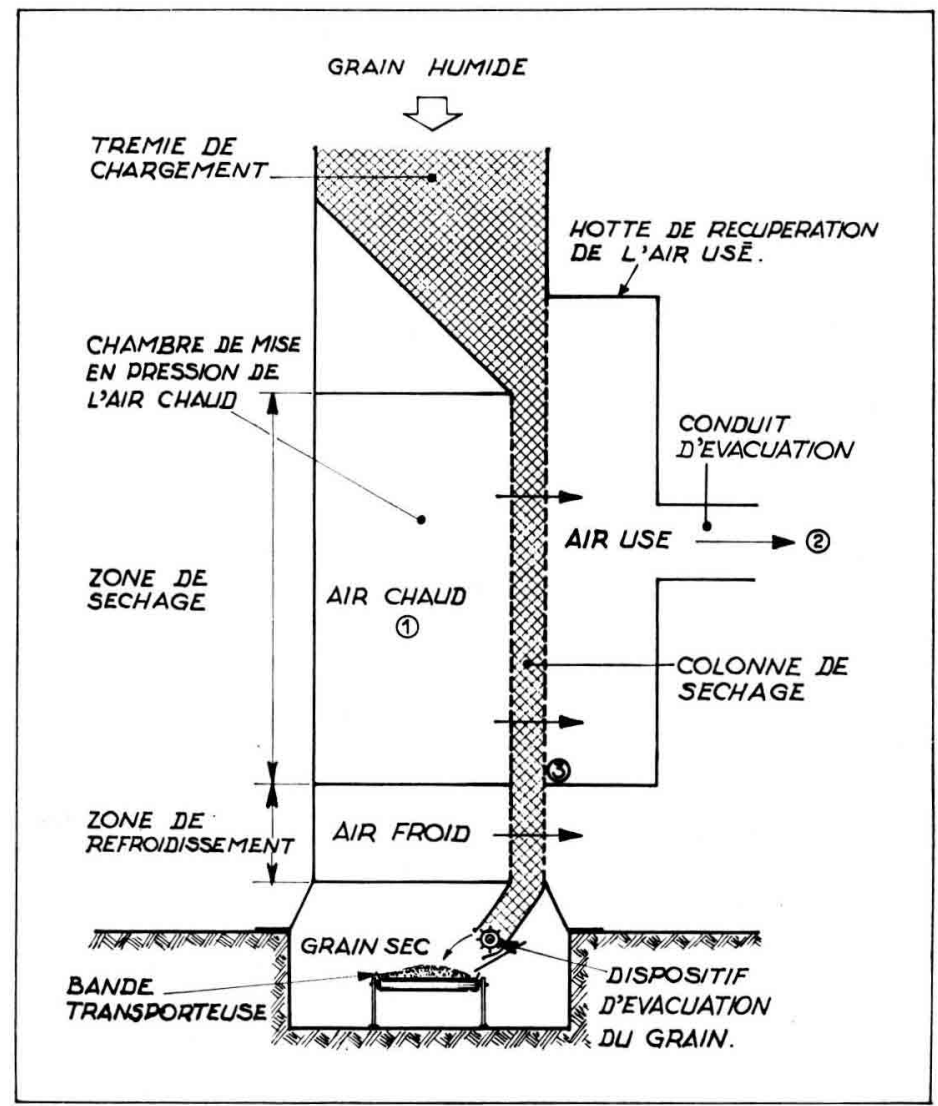

FIG. I. - Schema du séchoir continu

Emplacement de thermocouples (mesure des températures) :

(I) Température de l'air chaud;

(2) Température de l'air usé;

(3) Température de l'air usé en fin de séchage.

pouvant être maintenue constante pendant la durée de l'essai par une régulation automatique. Les débits d'air nécessaires au séchage et au refroidissement sont réglables au moyen d'un variateur de vitesse installé entre le moteur et le ventilateur. Le débit de grain séché, ainsi que l'humi- 
dité finale sont réglés par deux minuteries commandant, par périodes successives, le temps de fonctionnement du dispositif d'extraction et le temps d'arrêt. Enfin, le séchoir est équipé d'un doseur d'humidité en continu (type HYC) (') permettant de suivre et d'enregistrer l'humidité finale du grain séché.

III. - Mesures diverses

\section{a) Mesures des températures.}

Toutes les mesures de températures ont été effectuées par thermocouples et potentiomètres enregistreurs.

b) Mesure du débit d'air de séchage.

Celui-ci est déterminé par la méthode du "Caisson réduit " (Norme NF X ro.20o p. I9). L'organe de mesure est placé sur la conduite d'évacuation de l'air usé.

c) Mesure du débit de grain.

Il a été mesuré à l'aide de deux bascules automatiques placées en amont et en aval du séchoir.

d) Mesure de la teneur en eau du grain.

La teneur en eau, exprimée en pour cent de la substance humide (p. Ioo S. H.), a été mesurée suivant la méthode proposée par Guilвoт ( 1968 ) : étuvage à I $30^{\circ} \mathrm{C}$ d'un échantillon de Io grammes environ de grains de maïs entiers pendant $3^{8}$ heures $\left({ }^{2}\right)$.

\section{IV. - Description et signification pratique des traitements. Désignation et codification des échantillons et des lots}

Le protocole prévoyait 6 traitements, associant - suivant un plan factoriel — 3 modes de préstockage du maïs humide et 2 températures de séchage :

a) Modes de préstochage.

I $^{\circ}$ Préstochage de courte durée (20 à 30 heures) en cellule non ventilée ( $\mathrm{R}$ ).

Le délai séparant la récolte de la mise en cellule n'a pas excédé 8 heures : chargement du camion, transport, prénettoyage. Cette façon de procéder représente, à l'heure actuelle, ce que l'on peut faire de mieux pour réduire au minimum le temps s'écoulant entre la récolte et le séchage.

C'est pourquoi ce traitement sera désigné ainsi : "séchage immédiat après récolte », et le produit obtenu " maïs séché immédiatement après récolte ", symbolisé par la lettre " $\mathrm{R}$ ".

$2^{\circ}$ Préstockage de longue durée (6 jours), en cellule non ventilée (E).

Ce traitement représente un séjour prolongé du maîs humide, en boisseaux d'attente avant séchage, à la suite d'apports massifs de grain dans un organisme stockeur ne disposant pas, soit d'un séchoir de capacité suffisante, soit de cellules équipées d'une ventilation.

Des échauffements spontanés importants ont été constatés; aussi le traitement sera appelé : "échauffements" et le produit obtenu : " maïs échauffé ", symbolisé par la lettre "E ".

\section{$3^{\circ}$ Préstockage de très longue durée (45 jours) en cellule ventilée avec de l'air refroidi $(\mathrm{F})$.}

La technique utilisée pour réaliser ce traitement est celle choisie par certains pour faire face à l'augmentation et à l'irrégularité des quantités de maîs récolté : elle permet de constituer un volant de grain humide, et de le sécher ultérieurement. La durée de cet essai (un mois et demi) correspond aux campagnes de récolte les plus longues. Les conditions expérimentales sont les suivantes :

- débit d'air de renouvellement : Ioo $\mathrm{m}^{3} / \mathrm{h}$ et par $\mathrm{m}^{3}$ de grain ;

- température de l'air refroidi à la sortie du frigorifère : $5^{\circ} \mathrm{C}$;

- caractéristiques de l'air de ventilation :

(1) Appareil fabriqué par la Compagnie des Compteurs, 9 I-Massy.

(2) Proposition de normalisation faite à une réunion "Dosage de l'eau " de l'International Cereal Chemist, I968. 
- température : $7^{\circ} \mathrm{C}$ (réchauffage de $2^{\circ} \mathrm{C}$ dû au ventilateur et aux déperditions frigorifiques dans les gaines);

- hygrométrie : 85 à 90 p. Ioo d'humidité relative ;

- température de conservation du mais : entre 7 et $9^{\circ} \mathrm{C}$.

Les conditions étaient telles que, a priori, la conservation était supposée possible quant à la stabilisation de la température du grain (MATTEI 1969) mais assez risquée quant à l'évolution de la mycoflore (LAgrandeur et Poisson ig68).

Ce traitement sera ainsi désigné : conservation par ventilation d'air refroidi et le produit obtenu : maïs conservé par ventilation d'air refroidi, symbolisé par la lettre " $F$ 》。

\section{b) Température de stockage.}

Il s'agit de la température de l'air chaud, mis en contact avec le grain.

Io Température minima: $80^{\circ} \mathrm{C}$ régulée à $+2^{\circ} \mathrm{C}$, symbolisée par le nombre « 80 *.

$2^{\circ}$ Température maxima: $1_{4} 0^{\circ} \mathrm{C}$ régulée à $+5^{\circ} \mathrm{C}$, symbolisée par le nombre " $\mathbf{I}_{4} \mathrm{O}$ ".

Les températures de séchage choisies pour ces essais $\left(80\right.$ et $\mathrm{I}_{4} \mathrm{O}^{\circ} \mathrm{C}$ ) se situent aux extrêmes de la fourchette rencontrée dans la pratique, en ce qui concerne le maïs-grain destiné à l'alimentation animale (LASSERAN, 1969). Le schéma expérimental est factorialisé ; on aura toutefois une appréciation de l'effet simple dû aux températures sur les maïs \& $R$ »; sur les maïs " $E$ " et " $F$ ", on étudiera l'interaction préstockage-température.

c) Codification des échantillons et des lots.

Des échantillons ont été prélevés pour les analyses et tests de laboratoire à différents stades de l'évolution des traitements :

Stade I : échantillons prélevés après récolte au corn-sheller, au moment de la réception au C.N.E.E.M.A ; ces échantillons représentent le témoin de traitement pour chacun des 3 modes de préstockage. On distinguera donc : TTR, TTE, TTF, respectivement témoins de traitement de " $R$ ", " $E$ n et " $F$ ".

Stade II : échantillons prélevés après l'opération de préstockage, juste avant le séchage ; seuls les traitements " $E$ " et " $F$ » permettent d'obtenir des échantillons de ce stade. On les désignera par les symboles suivants « $E_{0}$ " et " $F_{0}$ ».

Stade III : échantillons prélevés après séchage et refroidissement ; on les désignera par un symbole associant celui du préstockage et celui de la température de séchage ; on aura ainsi la codification suivante :

$$
\mathrm{R}_{\mathbf{8 0}} ; \mathrm{E}_{\mathbf{8 0}} ; \mathrm{F}_{\mathbf{8 0}} ; \mathrm{R}_{\mathbf{1 4 0}} ; \mathrm{E}_{\mathbf{1 4 0}} ; \mathrm{F}_{\mathbf{1 4 0}} .
$$

Stade IV : échantillons prélevés après une conservation de 6 mois (décembre I 968 à mai I969). A ce stade, se situent en plus des échantillons pour certaines analyses, les lots destinés aux essais zootechniques. Pour ces derniers, on utilisera la notation symbolique du stade III, étant sous-entendu, bien sûr, et pour simplifier la rédaction, qu'il s'agit du stade IV.

Remarques : $\mathrm{I}^{\circ}$ Pour le traitement " $\mathrm{R}$ ", les stades I et II sont pratiquement confondus, étant donné la courte durée de préstockage.

$2^{\mathbf{0}}$ Les échantillons prélevés aux stades I et II ont été séchés dans un conditionneur de grain par ventilation énergique d'air ambiant, ou très peu réchauffé (au maximum de $5^{\circ} \mathrm{C}$ ) pendant 24 à 36 heures, sauf ceux destinés aux analyses microbiologiques qui ont été envoyés humides au laboratoire.

$3^{\circ}$ En plus des témoins de traitement, il existe un témoin de récolte (TR) obtenu après échantillonnage d'épis cueillis à la main quelques jours avant la récolte. Ces épis ont ensuite été séchés au conditionneur, puis égrenés; ce témoin de récolte est représentatif des potentialités du champ de maïs.

$4^{\circ}$ Pendant la phase de conservation de 6 mois, entre les stades III et IV, la température du grain a été maintenue en dessous de $10^{\circ} \mathrm{C}$ par une ventilation intermittente d'air froid ou refroidi. 


\section{RÉSULTATS DES ESSAIS - DISCUSSION}

\section{I. - Opérations de préstockage}

a) Échauffements (maïs « $\mathrm{E} »)$.

L'évolution de la température du grain en fonction du temps est représentée sur les figures 2 (maîs destiné au séchage à $80^{\circ} \mathrm{C}$ ) et 3 (mais destiné au séchage à $\mathrm{I} 40^{\circ} \mathrm{C}$ ). Une courte ventilation de 20 heures a été pratiquée le $3^{\mathrm{e}}$ jour pour égaliser les températures initiales, en raison du décalage des échauffements provoqué par le remplissage progressif des cellules. On note que la température du tiers supérieur de la cellule passe de 20 à $55^{\circ} \mathrm{C}$ en 2 jours et demi ( 60 heures); celle du tiers médian évolue moins rapidement; quant à celle du tiers inférieur, elle se stabilise entre $2 \mathrm{r}$

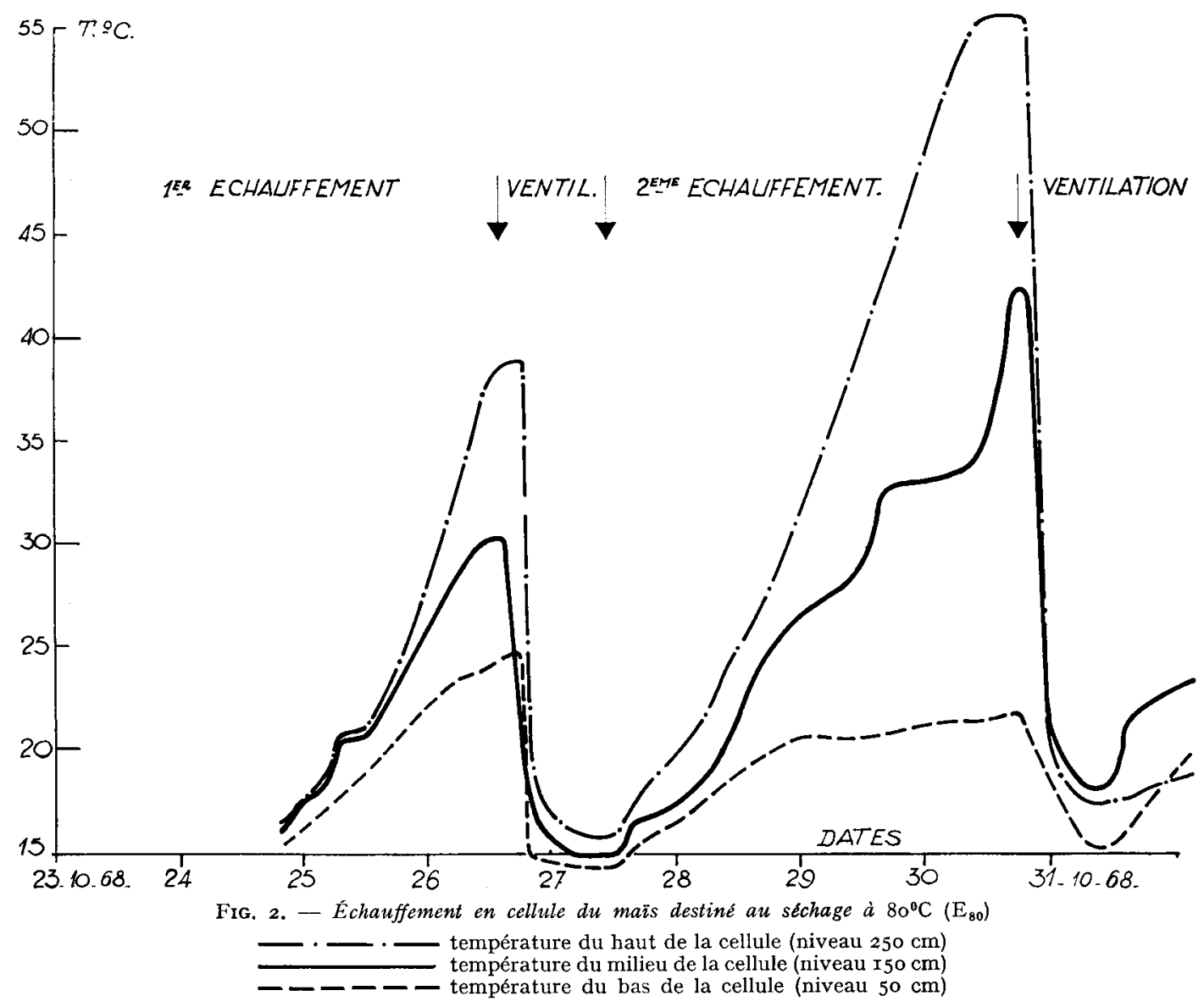

et $22^{\circ} \mathrm{C}$; on peut expliquer ce phénomène par l'accumulation du gaz carbonique dégagé par la respiration dans le fond des cellules et le blocage consécutif des mécanismes respiratoires du grain et des microorganismes. Les deux lots de maïs échauffé 
étaient donc hétérogènes au stade $\mathrm{n}^{0} \mathrm{II}$; toutefois on peut penser qu'à la suite des manipulations ultérieures (passage du grain au séchoir, nettoyage, ensachage) ces lots étaient suffisamment homogènes pour les essais zootechniques.

b) Conservation par ventilation d'air refroidi (maïs « $\mathrm{F} »)$.

Ce traitement a eu pour effets d'éviter les échauffements et de maintenir le grain en dessous de $9^{\circ} \mathrm{C}$ pendant 45 jours. Ces résultats confirment les travaux de MatTer (1969). La germination apparente a ainsi été évitée, toutefois on a noté la présence de tâches verdâtres de moisissures, observations qui recoupent celles de LAGRANDEUR et PoIsson ( 1968 ) : en effet, selon ces auteurs, Io jours suffiraient pour obtenir le demi-développement microbien sur du maïs à 35,2 p. Ioo de teneur en eau et ventilé avec de $1^{\prime}$ air à $5^{\circ} \mathrm{C}$, au débit de renouvellement de $150 \mathrm{~m}^{3} / \mathrm{h} / \mathrm{m}^{3}$ de maïs. De plus, à la vidange des cellules, on a constaté des prises en masse du grain ; les zones affectées par ce phénomène étaient les plus atteintes par les feutrages de myceliums. On peut penser que ces anomalies se sont produites dans des zones mal ventilées, à la suite de cheminements préférentiels de 1'air dans la masse de grain.

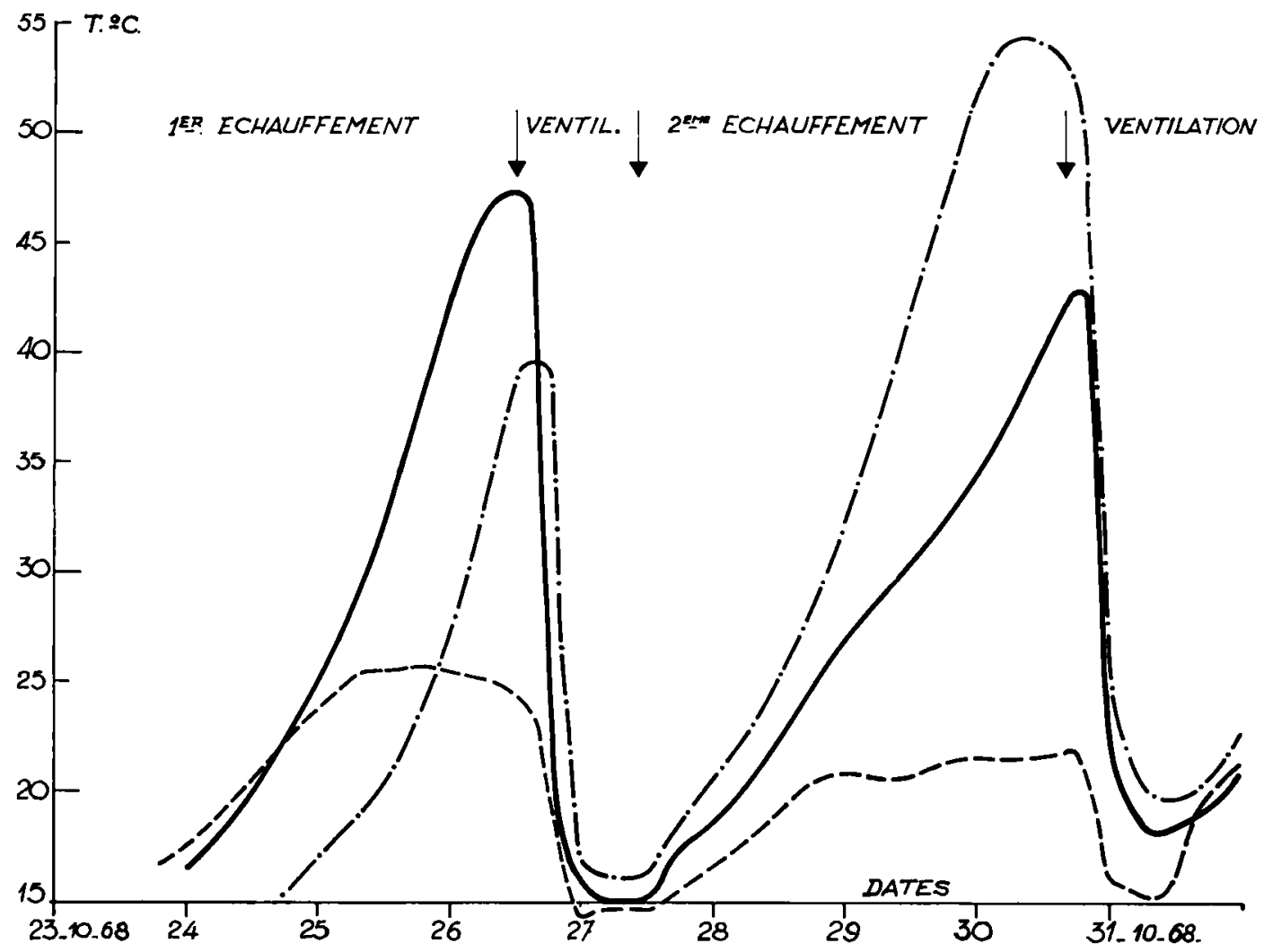

FIG. 3. - Échauffement en cellule du maïs destiné au séchage à $140^{\circ} \mathrm{C}\left(\mathrm{E}_{140}\right)$

- - - température du haut de la cellule (niveau $250 \mathrm{~cm}$ ) température du milieu de la cellule (niveau $150 \mathrm{~cm}$ )

- - - - température du bas de la cellule (niveau $50 \mathrm{~cm}$ ) 


\section{TABLEAU I}

Évolution de la teneur en eau du grain au cours des traitements

\begin{tabular}{c|c|c|c}
\hline & \multicolumn{3}{|c}{ Teneur en eau (\% S. H.) ${ }^{\left({ }^{1}\right)}$} \\
$\begin{array}{c}\text { Maïs destiné } \\
\text { aux } \\
\text { traitements }\end{array}$ & $\begin{array}{c}\text { Stade I } \\
\text { (réception) }\end{array}$ & $\begin{array}{c}\text { Stade II } \\
\text { (après préstockage } \\
\text { avant séchage) }\end{array}$ & $\begin{array}{c}\text { Stade III } \\
\text { (après séchage) }\end{array}$ \\
\cline { 2 - 4 } & & 35,5 & 14,2 \\
$\mathrm{R}_{\mathbf{8 0}}$ & 35,4 & 32,2 & 13,7 \\
$\mathrm{E}_{\mathbf{8 0}}$ & 35,1 & 28,0 & 13,6 \\
$\mathrm{~F}_{\mathbf{8 0}}$ & 35,8 & 35,8 & 12,9 \\
\hline $\mathrm{R}_{\mathbf{1 4 0}}$ & 35,0 & 31,7 & 13,5 \\
$\mathrm{E}_{\mathbf{1 4 0}}$ & 35,5 & 29,9 & 13,1 \\
$\mathrm{~F}_{\mathbf{1 4 0}}$ & & & \\
\hline
\end{tabular}

(1) S. H. = Substance Humide ou produit frais.

\section{II. - Opérations de séchage}

a) Évolution de la teneur en eau du grain au cours des traitements (tab1. I).

Les échauffements ont provoqué une baisse de teneur en eau de l'ordre de 3 p. roo. Cet abaissement est plus prononcé pour le traitement de conservation

TABLEAU 2

Évolution des températures moyennes de l'air pendant les opérations de séchage - Rendements de séchage

\begin{tabular}{|c|c|c|c|c|c|c|}
\hline \multirow{3}{*}{$\begin{array}{c}\text { Séchage } \\
\text { du } \\
\text { lot }\end{array}$} & \multicolumn{4}{|c|}{ Températures moyennes $\left({ }^{\circ} \mathrm{C}\right)$} & \multicolumn{2}{|c|}{$\begin{array}{c}\text { Rendements de séchage }(\mathbf{1}) \\
(\%)\end{array}$} \\
\hline & Air chaud & Air usé & $\begin{array}{l}\text { Air usé } \\
\text { saturé }\end{array}$ & $\begin{array}{l}\text { Air usé } \\
\text { en fin de } \\
\text { séchage }\end{array}$ & Global & $\begin{array}{l}\text { En fin de } \\
\text { séchage }\end{array}$ \\
\hline & 1 & 2 & 3 & 4 & 5 & 5 bis \\
\hline $\begin{array}{l}R_{80} \\
E_{80} \\
F_{80}\end{array}$ & $\begin{array}{l}80,1 \\
80,1 \\
80,2\end{array}$ & $\begin{array}{l}53,8 \\
55,2 \\
55,1\end{array}$ & $\begin{array}{l}31,4 \\
31,8 \\
30,0\end{array}$ & $\begin{array}{l}65,0 \\
65,4 \\
65,4\end{array}$ & $\begin{array}{l}54 \\
52 \\
50\end{array}$ & $\begin{array}{l}31 \\
30 \\
29\end{array}$ \\
\hline $\begin{array}{l}R_{140} \\
E_{140} \\
F_{140}\end{array}$ & $\begin{array}{l}139,9 \\
139,4 \\
140,4\end{array}$ & $\begin{array}{l}47,2 \\
48,9 \\
45,7\end{array}$ & $\begin{array}{l}41,0 \\
41,2 \\
40,3\end{array}$ & $\begin{array}{l}68,9 \\
70,1 \\
69,1\end{array}$ & $\begin{array}{l}94 \\
92 \\
95\end{array}$ & $\begin{array}{l}72 \\
71 \\
71\end{array}$ \\
\hline
\end{tabular}

(1) Rendements de séchage : Rendement global : $\frac{(1)-(2)}{(1)-(3)} \times 100$

Rendement en fin de séchage $: \frac{(1)-(4)}{(1)-(3)} \times 100$ 
par la ventilation d'air refroidi (6 à 7 p. IOo). Dans les deux cas, mais à des échelles différentes, la ventilation et la production de chaleur due aux phénomènes de respiration sont responsables de l'évaporation d'une partie de l'eau du maïs.

Les six lots de maîs ont été séchés à une teneur en eau relativement basse, comprise entre $\mathrm{I}_{3}$ et $\mathrm{I}_{4} \mathrm{p}$. Ioo ( $\mathrm{SH}$ ) et située au niveau du seuil de stabilisation (Guil,bot, Poisson, I963) pour éviter les effets secondaires dus à un stockage de plusieurs mois avant utilisation.

b) Évolution des températures moyennes de l'air pendant les opérations de séchage. Rendement de séchage (tabl. 2).

Io La température de l'air chaud mesurée dans la chambre de mise en pression (colonne $\mathrm{n}^{\mathrm{o}} \mathrm{I}$ ) est très proche de la température de référence.

$2^{\circ}$ La température moyenne de l'air usé mesurée dans la conduite d'évacuation (colonne $\mathrm{n}^{\circ}$ 2) est paradoxalement plus basse pour les séchages à $140^{\circ} \mathrm{C}$; ceci est lié au rendement de séchage (colonne no 5 ).

$3^{\circ}$ La température de bulbe humide de l'air chaud, obtenue par lecture du diagramme de l'air humide est mentionnée à la colonne $\mathrm{n}^{0} 3$ sous l'appellation de " température de l'air usé saturé ". KNEULE (I964) la définit aussi comme étant la température limite de refroidissement, obtenue pour un rendement théorique de séchage de Ioo p. Ioo.

$4^{0}$ Dans la colonne $n^{0} 4$ est indiquée la température de l'air usé en fin de séchage que 1'on a mesurée au bas de la zone de séchage. Cette température est plus élevée que la température moyenne de l'air usé : dans les dernières phases de l'opération de séchage, la vitesse d'évaporation est ralentie de façon progressive en raison de la baisse de diffusion de l'eau. L'effet évaporatoire étant moins intense au cours de cette phase, la température de l'air usé augmente ; il en est de même pour la température de surface des grains ; ceux-ci peuvent subir une torréfaction partielle superficielle lorsque le péricarpe, surséché par rapport à l'intérieur de l'amande, subit des températures trop élevées. Nous ne connaissons pas la température de début de torréfaction; de plus, au cours des essais, il ne nous a pas été possible de mesurer la température du grain en fin de séchage. Nous avons seulement constaté une proportion importante de grains brûlés après les séchages à $140^{\circ} \mathrm{C}$. Toutefois, il faut éviter de généraliser à tous les séchoirs, et souligner que les conditions expérimentales sont autant responsables de cet incident que la température de $140^{\circ} \mathrm{C}$; en effet dans la colonne de séchage, les couches de grain ne se mélangent pas lors de leur descente; il y a donc un surséchage pour celles placées du côté de la chambre de mise en pression de l'air chaud ; par ailleurs, le phénomène a encore été accentué par le fait que l'humidité finale moyenne a été volontairement abaissée en dessous des normes commerciales.

$5^{\circ}$ Les colonnes 5 et 5 bis présentent les rendements de séchage : rendement global (ou moyen) et rendement de fin de séchage. La formule utilisée pour les calculer est celle proposée par AGUilar et Boyce (I966). On constate une amélioration considérable de ceux-ci pour les séchages à $\mathrm{I}_{40^{\circ}} \mathrm{C}$; pour les deux modes de séchage, on note une diminution du rendement de fin de séchage.

c) Évolution des caractéristiques de séchage (tab1. 3).

Io Les débits spécifiques d'air chaud (colonnes I et I bis) sensiblement constants pour un même mode de séchage ont du être abaissés dans le second afin de permettre 
une montée de la température jusqu'à $140^{\circ} \mathrm{C}$. Par débit spécifique, volumique ou massique, on désigne le débit rapporté à l'unité de volume de grain compris dans la zone de séchage.

$2^{0}$ La puissance thermique utile (colonne $\mathrm{n}^{0}$ 2) a été maintenue constante pour un même mode de séchage. Il s'agit de la puissance thermique effectivement utilisée pour le séchage du grain, obtenue à partir du débit masse d'air et de la différence enthalpique entre l'air chaud et l'air ambiant extérieur considéré à $20^{\circ} \mathrm{C}$.

$3^{\circ}$ La puissance d'évaporation (colonne $n^{0} 3$ ) est obtenue par le calcul à partir des mesures de débit et de teneur en eau du grain (BARTHELEMY, LASSERAN, Ig66). Malgré une réduction du débit d'air de l'ordre de $30 \mathrm{p}$. Ioo pour les séchages à $\mathrm{I} 40^{\circ} \mathrm{C}$,

TABLEAU 3

Évolution des caractéristiques de séchage

\begin{tabular}{|c|c|c|c|c|c|c|}
\hline \multirow{4}{*}{$\begin{array}{l}\text { Séchage } \\
\text { du } \\
\text { lot }\end{array}$} & \multicolumn{2}{|c|}{$\begin{array}{l}\text { Débits spécifiques de } \\
\text { l'air chaud }\end{array}$} & \multirow{2}{*}{$\begin{array}{c}\text { Puissance } \\
\text { thermique } \\
\text { utile }\end{array}$} & \multirow{2}{*}{$\begin{array}{c}\text { Puissance } \\
\text { d'évaporation }\end{array}$} & \multirow{2}{*}{$\begin{array}{l}\text { Consommation } \\
\text { thermique } \\
\text { massique }\end{array}$} & \multirow{2}{*}{$\begin{array}{c}\text { Temps } \\
\text { moyen de } \\
\text { séchage }\end{array}$} \\
\hline & Volumique ( $\left.{ }^{(}\right)$ & Massique & & & & \\
\hline & $\left(\mathrm{m}^{3} / \mathrm{h} \cdot \mathrm{m}^{3}\right)$ & $\left(\mathrm{kg} / \mathrm{h} \cdot \mathrm{m}^{3}\right)$ & $(\mathrm{kcal} / \mathrm{h})$ & $(\mathrm{kg} / \mathrm{h})$ & (kcal/kg) & $(\mathrm{mm})$ \\
\hline & 1 & 1 bis & 2 & 3 & 4 & 5 \\
\hline $\mathrm{R}_{80}$ & 5058 & 6119 & 86398 & 87,2 & 991 & 163 \\
\hline$E_{80}$ & 5049 & 6108 & 86407 & 79,6 & 1085 & 148 \\
\hline $\mathrm{F}_{80}^{\circ 0}$ & 5085 & 6153 & 86522 & 70,9 & 1220 & 122 \\
\hline $\mathrm{R}_{\mathbf{1 4 0}}$ & 3470 & 4198 & 118549 & 152,1 & 779 & 101 \\
\hline $\mathrm{E}_{140}$ & 3476 & 4205 & 118290 & 146,3 & 808 & 76 \\
\hline$F_{140}$ & 3519 & 4158 & 119976 & 155,2 & 773 & 66 \\
\hline
\end{tabular}

(1) Débit spécifique volume corrigé à $20^{\circ} \mathrm{C}$ et 75 p. 100 d'humidité relative (masse volumique : 1,2098 $\mathrm{kg}$ d'air sec par $\mathrm{m}^{3} \mathrm{~d}^{\prime}$ air humide).

elle subit, à cette température, une augmentation très importante d'environ $90 \mathrm{p}$. Ioo.

Le maintien du même débit d'air lors des essais à $140^{\circ} \mathrm{C}$ aurait permis un accroissement de la puissance d'évaporation de l'ordre de IIo à I20 p. Ioo. Le débit de grain séché aurait varié dans les mêmes proportions. A noter encore que pour les séchages à $80^{\circ} \mathrm{C}$, la puissance d'évaporation est affectée, soit par la teneur en eau du grain avant séchage (tabl. I), soit par le traitement de préstockage.

$4^{0}$ La consommation thermique massique (colonne $n^{0} 4$ ) est le rapport de la puissance thermique utile à la puissance d'évaporation. Cette caractéristique est une composante du prix de revient du séchage. Elle est notablement influencée par la température de l'air chaud, comme les rendements de séchage. A $80^{\circ} \mathrm{C}$, son évolution est inverse de celle de la puissance d'évaporation.

$5^{0}$ Enfin, dans la colonne $n^{\circ} 5$, est mentionné le temps moyen de séchage (temps moyen de séjour du grain dans la zone de séchage). Il est beaucoup plus court à $\mathrm{I}_{4} \mathrm{O}^{\circ} \mathrm{C}$ qu'à $80^{\circ} \mathrm{C}$ et, pour un même mode de séchage, il augmente lorsque la teneur en eau du grain avant séchage est plus élevée. 


\section{CONCLUSIONS}

Les 6 traitements réalisés sur le maïs-grain sont loin de représenter toutes les combinaisons possibles des modes de préstockage avec les températures de séchage. Toutefois, ces essais doivent apporter des renseignements utiles sur les principales erreurs à éviter et sur la nature des traitements expérimentés puisqu'ils associent méthodes courantes et conditions extrêmes, travail soigné et pratiques déconseillables.

Les essais de séchage ont permis de confirmer ou de préciser certains points :

- 1'échauffement du maïs-grain humide en cellule est extrêmement rapide : le temps de demi-échauffement, pour une élévation de la température du grain de $\mathrm{r}_{5}$ à $35^{\circ} \mathrm{C}$, est de 40 heures ;

- la conservation par ventilation d'air refroidi est un procédé qui permet de stabiliser la température du grain, sans pour autant éliminer les développements de moisissures si la durée de stockage est trop longue;

- le séchage en un seul passage du maïs très humide s'accompagne d'une baisse de rendement lors de la phase finale de séchage, en raison de la baisse de diffusion de l'eau interne;

- le séchage s'effectue dans de meilleures conditions à haute température, d'un point de vue, à la fois thermodynamique (augmentation du rendement de séchage, abaissement de la consommation thermique massique) et quantitatif (accroissement du débit de grain, diminution du temps de séchage), ce qui est un avantage économique important dans la mesure où celui-ci n'est pas annulé par des pertes de qualité affectant la valeur commerciale, alimentaire ou technologique du maîs.

\section{SUMMARY}

\section{I.- METHODS FOR PREPARATION OF THE SAMPLES}

A Study on "the influence of conditioning method in the maize quality " was undertaken in 1968. Six treatments were carried out under well-established different conditions, bearing in mind some of them are the good ones and some of them not.

Three methods of wet-maize preliminary storage have been worked out before drying.

Io Short stay in bin of less than 30 hours, say an immediate drying after harvest (R).

$2^{\circ}$ Long stay in bin of 6 days having created a spontaneous heating (E).

$3^{\circ}$ In-bin storage during 45 days with cooling air by means of a refrigerating plant (F).

Drying processus were effected in one passing through a continuous dryer at the following temperatures :

a) $80^{\circ} \mathrm{C}$, temperature regarded as moderate $(80)$;

b) $140^{\circ} \mathrm{C}$, temperature regarded as excessive ( 140 ).

The whole measures carried out during tests allow to establish some numerical data relating to the drying method employed. The main results obtained of this study are as follow : drying yield, mass heat consumption, drying period and drier outflow rate are considerably bettered when the drying process is carried out at high temperature.

After these treatments, experts on grain trading and maize starch industries, biochemical research workers, microbiologists and animal scientists are liable to give their opinion on quality of maize obtained thuswise. 


\section{RÉFÉRENCES BIBLIOGRAPHIQUES}

Aguilar C. S., Boyce D. S., I966. Temperature ratios for measuring efficiency and for the control of driers. Agric. Eng. Res., 11, I9-23.

Barthélémy P., Lasseran J. C., I966. Où en sont les constructeurs de séchoirs continus pour céréales ? Le producteur agricole français, 153, 9-1o et 154, 9-10.

Cabell C. A., Davis R. E., Saul K., I958. Some effects of variation in drying temperature, heating time, air-flow rate, and moisture content on nutritive value of field shelled corn. J. Anim. Sci., 17, I204.

Calet C., Mercier C., I966 : Influence des conditions de production, de récolte et de stockage sur la composition biochimique des céréales; répercussions des variations sur la valeur nutritionnelle. $A n n$. Nutr. Alim., 20, 24I-256.

Guilbot A., Poisson J., I963. Conditions de stockage et durée de conservation des grains. Journées d'études sur la conservation des grains (I. T. C F -C.E. R. D. I. A.-C. N. E. E. M. A.). Supplément du Producteur agricole français, I5-27.

Hathaway I. L., Yung F. D., Kiesselbach T. A., I952. The effect of drying temperature upon the nutritive value and commercial grade of corn. J. A nim. Sci., 11, 430-44o.

Jouın C., 1957. Le froid est-il un moyen de conservation et de séchage du grain? Génie rural, 12 , 489-490.

Jovin C., I96r. Échanges d'eau et de chaleur entre l'air et le grain. Refroidissement par la ventilation. Bull. E. F. M., 185, 247-253.

Journ C., I965. Le froid et la conservation des céréales. Bull. E. F, M., 205, 9-13.

Journ C., I966a) L'emploi de l'air froid pour la conservation des grains. Bull. Inf. C. N. E. E. M. A., 106, $5^{\mathrm{e}}$ partie.

Jouin C., I966 b) Coût des chaînes d'équipement et qualité du maïs. La récolte et le séchage du maïs. Journées Internationales de récolte et de séchage du maîs, à Membrolles (Loir-et-Cher). Doc. I. T. C. F.A. G. P. M., 75-82,

Kneule F., 1964. Le Séchage. Eyrolles Ed. Paris, 75-78.

Lagrandeur G., Poisson J., I968. La microflore du maïs, son évolution en fonction des conditions hydriques et thermiques de stockage en atmosphère renouvelée. Indus. Alim. Agr., 6-7, 775-788.

LASSERAN J. C., I969. Le séchage des grains par air chaud. Le producteur agricole français, 46, I3-I5.

MAQuet E., I966. Solutions économiques et pratiques de récolte et de conservation du maïs. La récolte et le séchage du maïs. Journées Internationales de récolte et de séchage du maïs à Membrolles (Loir-et-Cher). Doc. I. T. C. F.-A. G. P. M., 83-94.

Mattei J. N., I969. a) Données techniques sur la ventilation des grains. C. N. E. E. M. A., Étude, 329.

Mattei J. N., ig69. b) Conservation du grain par le froid. Bull. Inf. C. N. E. E. M. A., 137, 36-45.

Milner C. K., Woodforde J., I965. The effect of heat in drying on the nutritive value of wheat for animal feed. J. Sci. Food Agric., 16, 369-373. 